\title{
Solar-Wind Bulk Velocity Throughout the Inner Heliosphere from Multi-Spacecraft Measurements
}

\author{
A. Opitz $\cdot$ A. Fedorov $\cdot$ P. Wurz $\cdot$ K. Szego • \\ J.-A. Sauvaud · R. Karrer • A.B. Galvin · S. Barabash • \\ F. Ipavich
}

Received: 5 February 2010 / Accepted: 21 May 2010 / Published online: 18 June 2010

(C) The Author(s) 2010. This article is published with open access at Springerlink.com

\begin{abstract}
We extrapolate solar-wind bulk velocity measurements for different in-ecliptic heliospheric positions by calculating the theoretical time lag between the locations. The solar-wind bulk velocity dataset is obtained from in-situ plasma measurements by STEREO A and B, SOHO, Venus Express, and Mars Express. During their simultaneous measurements between 2007 and 2009 we find typical solar activity minimum conditions. In order to validate our extrapolations of the STEREO A and B data, we compare them with simultaneous in-situ observations from the other spacecraft. This way of cross-calibration we obtain a measure for the goodness of our extrapolations over different heliospheric distances. We find that a reliable solar-wind dataset can be provided in case of a longitudinal separation less than 65 degrees. Moreover, we find that the time lag method assuming constant velocity is a good basis to extrapolate from measurements in Earth orbit to Venus or to Mars. These extrapolations might serve as a good solar-wind input information for planetary studies of magnetospheric and ionospheric processes. We additionally show how the stream-stream interactions in the ecliptic alter the bulk velocity during radial propagation.
\end{abstract}

\footnotetext{
A. Opitz $(\bowtie) \cdot$ A. Fedorov $\cdot$ J.-A. Sauvaud

Centre d'Etude Spatiale des Rayonnements (CNRS-UPS), University of Toulouse, Toulouse, France e-mail: opitz@cesr.fr

P. Wurz · R. Karrer

Department of Space Science and Planetology, Physics Institute, University of Bern, Bern, Switzerland
}

K. Szego

KFKI Research Institute for Particle and Nuclear Physics, Budapest, Hungary

A.B. Galvin

Space Science Center, University of New Hampshire, Durham, USA

S. Barabash

Swedish Institute of Space Physics, Kiruna, Sweden

F. Ipavich

Department of Physics and Astronomy and IPST, University of Maryland, College Park, USA 


\section{Motivation}

The solar-wind plasma properties directly influence the size and shape of the planetary bow shock and magnetopause, as well as define the magnetosheath characteristics. Their variation might have several effects, for instance on the planetary ion escape (Lundin et al., 2008). Hence, we make efforts to provide a full-time solar-wind dataset to support studies by planetary missions. These spacecraft are traversing the different plasma regions in the planetary environment, as a result they provide valuable measurements in the solar wind, magnetosheath and magnetosphere. However, one single spacecraft cannot provide simultaneous in-situ measurements in the different plasma regions. We try to fill up such data gaps of the local solar-wind plasma measurements by extrapolating datasets measured continuously by solar missions.

It was found that the bulk velocity of the solar wind ejected from the same source location on the solar surface but at different times is persistent at least up to two days (Opitz et al., 2009). This two-day period is derived from a time lag of $\sim 30$ degree longitudinal spacecraft separation. From the proton bulk velocity results of very good correlation we extrapolate that even in $\sim 5$ days the correlation coefficient must be still higher than 0.5 , so the solar wind can be considered as more or less persistent. As a result, in this paper we extend our solar-wind bulk velocity investigations with cases above 30 degree longitudinal separation.

We note that this study is performed during solar activity minimum, when transient events such as coronal mass ejections are rare. Such events would modify the plasma properties so that the measured in-situ parameters could not provide a good input for our extrapolations.

\section{Plasma Datasets}

Our solar-wind bulk velocity dataset is obtained from plasma measurements by five spacecraft: The Solar Terrestrial Relations Observatory (STEREO) $A$ and $B$ spacecraft carry the Plasma and Suprathermal Ion Composition (PLASTIC) experiment (Galvin et al., 2008). The Solar and Heliospheric Observatory (SOHO) spacecraft accommodates as part of the Charge Element and Isotope Analysis System (CELIAS) Mass Time-of-Flight Spectrometer (MTOF) the Proton Monitor (PM) investigation (Hovestadt et al., 1995; Ipavich et al., 1998). The Venus Express (VEX) spacecraft carries as part of the ASPERA-4 plasma instrument package the Ion Mass Analyzer (IMA) experiment (Barabash et al., 2007) and the Mars Express (MEX) spacecraft accommodates as part of the ASPERA-3 plasma instrument package the Ion Mass Analyzer (IMA) investigation (Barabash et al., 2006). We obtain the solar-wind bulk velocity dataset from these five spacecraft measurements. The two PLASTIC instruments provide one-minute and the PM one-hour measurements as long as the VEX and MEX datasets seem to be rather sporadic since they cross frequently the planetary bow shock, hence we had to select plasma measurements for periods where the spacecraft were upstream of the respective bow shock to assure a pure solar-wind dataset. During these simultaneous measurements between 2007 and 2009 we find the usual solar activity minimum conditions with the typical alternating slow and fast solar-wind stream pattern.

\section{Method, Prediction and Its Validation}

We extrapolate solar-wind bulk velocity measurements for different heliospheric positions by time shifting the measured dataset with the theoretical time lag between the spacecraft 
and the target position. The applied method is described in detail by Opitz et al. (2009), where they calculate the time lag between the STEREO A and B spacecraft by separating the effect of the radial and longitudinal spacecraft separation.

The time lag from the radial separation is calculated by assuming constant bulk velocity of the solar wind along the radial propagation direction, which is acceptable beyond reasonable distances from the solar surface. Note, however, that larger radial distances are affected by the acceleration and deceleration of the bulk plasma due to fast and slow stream interactions. As an example, such large-scale heliospheric radial distances were studied by Burlaga et al. (2008), where they compared one-dimensional time-dependent MHD model results with in-situ Voyager 2 measurements. In this paper we study how far our simple approximation is valid on somewhat shorter distances.

The time lag from the longitudinal separation $\left(t_{r}\right)$ is calculated from the solar rotation rate. In this study we perform the time lag calculation in the same way but between the measuring spacecraft and the target position. To extrapolate the solar-wind properties to a given heliospheric position, the measured dataset is time shifted by this theoretical time lag.

In order to validate our extrapolations, we compare them with in-situ solar-wind plasma measurements. This way we obtain the validity of our extrapolation of the solar-wind bulk velocity over different heliospheric distances. We additionally define a goodness index (GI) from the longitudinal time lag as GI $=\left(\mathrm{CR} / 2-\left|t_{r}\right|\right) /(\mathrm{CR} / 2) \cdot 100$, where CR stands for Carrington Rotation (27.2753 day). This goodness index is meant to describe the expected accuracy of our prediction. The goodness of the prediction depends on the heliospheric constellation: it is supposed to be good if the longitudinal time lag is less than five days (GI $>\sim 63$ ), and the radial time lag is less than the characteristic time in which the streamstream interactions might significantly alter the solar-wind bulk velocity. This characteristic time depends on several factors such as the occurrence and heliospheric position (distance and latitude) of these stream interfaces, the velocity difference between the two streams, the density or the magnetic field configuration.

\section{Results and Discussion}

In order to predict the solar-wind properties at a given heliospheric position, we time shift both datasets measured by the two STEREO spacecraft. After adjusting for the time lag between the spacecraft and the target position, we obtain two predictions for the target position.

In August 2007 planet Venus passed in front of both STEREO spacecraft and Mars was about 60 degrees in longitude from Earth (Figure 1). This heliospheric constellation was a good opportunity to cross-calibrate the solar-wind plasma measurements of the five spacecraft and to assess the validity region of our predictions.

We extrapolated solar-wind bulk velocity measurements performed by STEREO to the locations of Venus, Earth and Mars. These predictions were compared with the in-situ measurements by VEX, SOHO and MEX spacecraft, respectively (Figure 2). We included in the plots the correlation coefficients between the prediction and in-situ measurements for the investigated time period. As expected, the smaller spacecraft separations resulted in higher correlations and the poorest correlation of 0.671 was found between MEX and STEREO B since they have the largest separation. Additionally, the goodness, GI, is shown in the plots, which indicates here that we expect a good prediction for the investigated time period. Indeed, the correlation coefficients are high (between 0.671 and 0.911 ), the predictions agree well with the in-situ measurements for most of the time. A significant exception is the special period of DOY 227-235 in 2007, where an especially fast evolution of the solar wind 

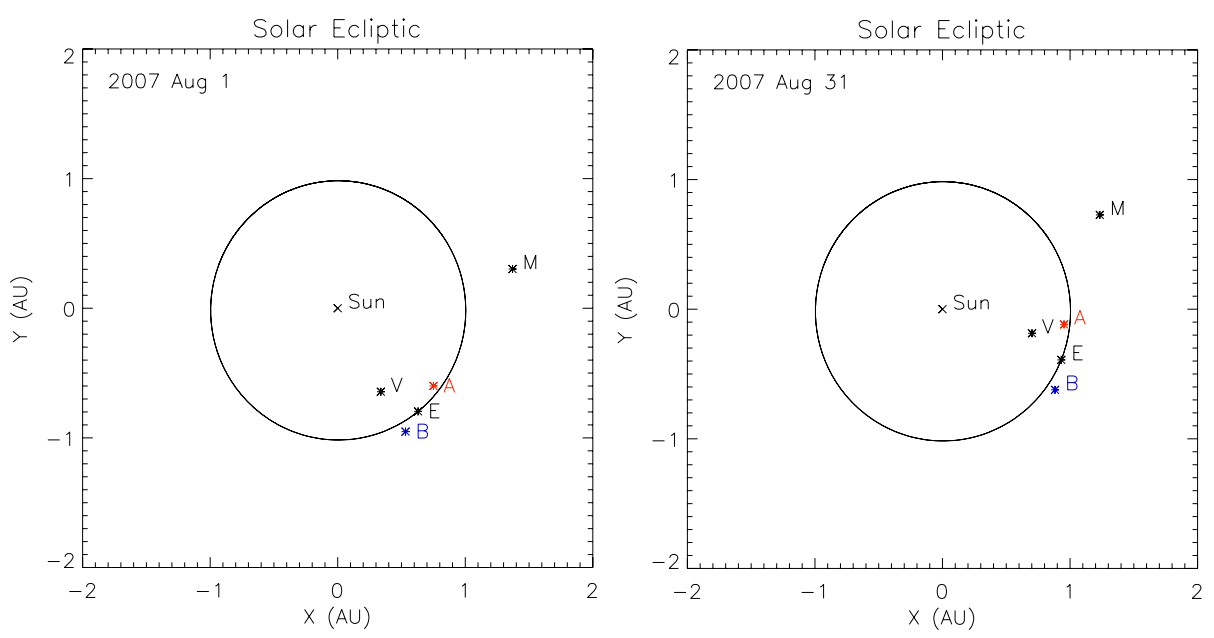

Figure 1 In-ecliptic position of Earth (E), Venus (V), Mars (M), STEREO A (red) and B (blue) on 1st August 2007 (left) and 31st August 2007 (right).

was followed by a latitudinal effect as discussed already in Opitz et al. (2009). Neglecting this time period and further analyzing the results, it is visible that the Venus prediction is tilted slightly to the right, while the Mars prediction is clearly tilted slightly to the left relative to the in-situ measurements. In fact, during radial propagation in the ecliptic the fast solar wind tries to overtake the slow solar wind, what triggers acceleration and deceleration of the bulk plasma. As Figure 2 shows, the plasma must have been accelerated in the front and decelerated in the back of the stream interface during its radial propagation. The tilt in the predictions relative to the in-situ measurements is due to the omission of this acceleration-deceleration process by assuming constant solar-wind bulk velocity. At the fast and slow solar-wind interaction the density increases and forms a compression region, the frozen-in magnetic field is stressed, hence pressure gradients form. The above shown bulk plasma acceleration and deceleration is due to these effects, which is accounted for in models, for instance by MHD simulation (see Zieger and Hansen, 2008 and references therein). In this paper we just test the limitations of the simple method that assumes solarwind propagation by constant velocity. We find that these predictions are valid from Earth orbit to Venus $(0.7 \mathrm{AU})$ or to Mars orbit $(\sim 1.5 \mathrm{AU})$, but the in-ecliptic extrapolation to the solar surface (backmapping) using the constant velocity assumption results in significantly overtilted curves.

\section{Conclusions}

We conclude that even a simple model of solar-wind propagation can predict the solar-wind bulk velocity between Venus and Mars orbits if the longitudinal separation between the target position and the solar-wind measuring spacecraft is not larger than about 65 degrees. We have additionally shown that the stream-stream interactions alter the bulk velocity during radial propagation. There is acceleration in front of the stream interface, while deceleration behind, which is already visible when extrapolating from Earth to Mars or to Venus orbit. We believe though, that this modification is still on a tolerable level in this region, but beyond these radial distances a more complex model is needed. 
Figure 2 Predicted and measured solar-wind bulk velocity at Venus (top), Earth (center) and Mars (bottom) in August 2007. Black crosses denote the in-situ measurements by VEX, SOHO and MEX, respectively. All predictions used PLASTIC measurements onboard STEREO A (red) and B (blue). The correlation coefficient between prediction and in-situ measurement is also given. The goodness index (between 0 and 100) is shown at the bottom of each plot, where red corresponds to STEREO A and blue to STEREO B. Above the dashed line we expect a good prediction since the spacecraft longitudinal separation is lower than 65 degrees.
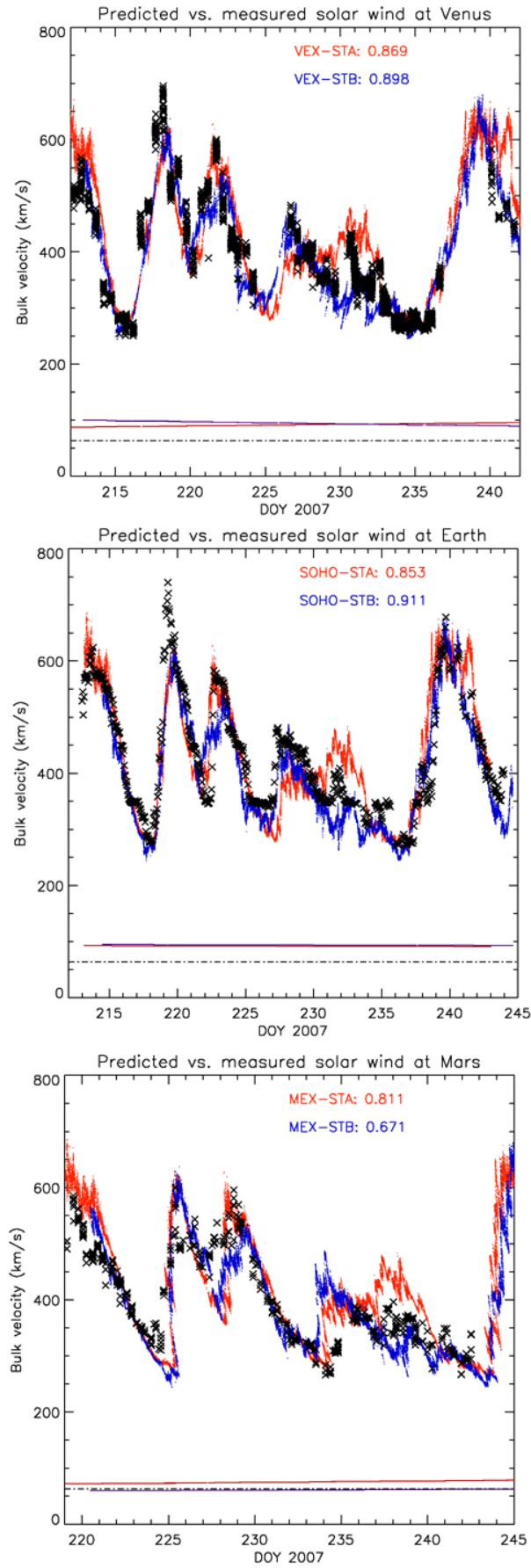
In the above given limitations we can provide a full-time (one-minute) solar-wind bulk velocity dataset for 2007-2009 for any heliospheric positions. These predictions can be improved by more sophisticated in-ecliptic radial solar-wind propagation models and further solar-wind temporal evolution results obtained at greater STEREO longitudinal spacecraft separations.

Acknowledgements The authors acknowledge contribution from the STEREO, SOHO, VEX and MEX groups. They also thank for discussions with Rickard Lundin and Bertalan Zieger. This work was supported by a CNES grant for CESR (CNRS-UPS).

Open Access This article is distributed under the terms of the Creative Commons Attribution Noncommercial License which permits any noncommercial use, distribution, and reproduction in any medium, provided the original author(s) and source are credited.

\section{References}

Barabash, S., Lundin, R., Andersson, H., Brinkfeldt, K., Grigoriev, A., Gunell, H., Holmstrom, M., Yamauchi, M., Asamura, K., Bochsler, P., et al.: 2006, Space Sci. Rev. 126, 113.

Barabash, S., Sauvaud, J.-A., Gunell, H., Andersson, H., Grigoriev, A., Brinkfeldt, K., Holmstrom, M., Lundin, R., Yamauchi, M., Asamura, K., et al.: 2007, Planet. Space Sci. 55, 1772.

Burlaga, L.F., Ness, N.F., Acuna, M.H., Wang, Y.-M., Sheeley, N.R. Jr., Wang, C., Richardson, J.D.: 2008, J. Geophys. Res. 113, A02104.

Galvin, A.B., Kistler, L.M., Popecki, M., Farrugia, C.J., Simunac, K.D.C., Ellis, L., Mobius, E., Lee, M.A., Boehm, M., Carroll, J., et al.: 2008, Space Sci. Rev. 136, 437.

Hovestadt, D., Hilchenbach, M., Burgi, A., Klecker, B., Laeverenz, P., Scholer, M., Grunwaldt, H., Axford, W.I., Livi, S., Marsch, E., et al.: 1995, Solar Phys. 162, 441.

Ipavich, F.M., Galvin, A.B., Lasley, S.E., Paquette, J.A., Hefti, S., Reiche, K.-U., Coplan, M.A., Gloeckler, G., Bochsler, P., Hovestadt, D., et al.: 1998, J. Geophys. Res. 103(A8), 17205.

Lundin, R., Barabash, S., Fedorov, A., Holmström, M., Nilsson, H., Sauvaud, J.-A., Yamauchi, M.: 2008, Geophys. Res. Let. 35, L09203.

Opitz, A., Karrer, R., Wurz, P., Galvin, A.B., Bochsler, P., Blush, L.M., Daoudi, H., Ellis, L., Farrugia, C.J., Giammanco, C., et al.: 2009, Solar Phys. 256, 365.

Zieger, B., Hansen, K.C.: 2008, J. Geophys. Res. 113, A08107. 